\title{
An Intelligent Health Monitoring System based on Secure Distributed Routing in Wireless Body Area Networks
}

\author{
Rajeev Sharma ${ }^{1}$, Dr. Sandeep Singh Kang ${ }^{2}$ \\ ${ }^{1}$ Research Scholar, Department of Computer Science \& Engineering, Chandigarh University, Gharuan, Mohali, India \\ cecm.cse.rajeev@gmail.com \\ ${ }^{2}$ Professor, Department of Computer Science \& Engineering, Chandigarh University, Gharuan, Mohali, India \\ sandeepkang.cse@ cumail.in
}

\begin{abstract}
Security analysis in Wireless Body Area Network (WBAN) is a case study remote health monitoring as after measure for deploying security and routing features is introduced in this research paper. This research work is focused on the security factor of WBAN technology during the sensed data packet transmission by a sensor node from the patient body using the routing protocol. An Intelligent Health Monitoring (IHM) system based on Secure Distributed and Energy Efficient Routing (SDEER) protocol in WBAN technology using the Feed Forward Back Propagation Neural Network (F2-BPNN) with Particle Swarm Optimization (PSO) as an optimization algorithm is proposed in this research to solve out the security constraints. This experimental study designed and develop a simulator of WBAN using the MATLAB R2016a Software with artificial intelligence toolbox and optimization toolboxes. We have considered the three-tier communication in the IHM system such as Intra-WBAN communication, Inter-WBAN communication, and Beyond-WBAN communication using the SDEER protocol. Where F2BPNN technique used to select cluster heads or relays or intermediate sensor nodes based on their residual energy and distance from the receiver node. When the evaluation parameters of proposed IHM system is compared with a few other state-of-art methods, the proposed routing protocol achieves the best performance in terms of throughput, energy consumption, and queue size and it is observed that proposed IHM system is significantly prolonged the network lifetime by reducing the energy consumption rate.
\end{abstract}

Key words: Wireless Body Area Network (WBAN), Intelligent Health Monitoring (IHM), Secure Distributed and Energy Efficient Routing (SDEER), Constrained Markov Decision Processes (CMDP), Feed Forward Back Propagation Neural Network (F2BPNN), Particle Swarm Optimization (PSO)

\section{INTRODUCTION}

Wireless sensor network (WSN) is a network that consists of tiny sensor nodes that are powered by the battery and have limited resources of transmission power, the bandwidth of the network, and computation power. Each sensor node in WSN monitors and collects the physical environmental information [1]. WSN is an emerging field that its applications are multifold and can be found in many areas such as battlefield monitoring, environment monitoring, health monitoring, agriculture field monitoring, and smart home. Nowadays, WSN based health monitoring applications have their own impact to monitor the patients by informing the doctors and patients guardians. Wireless body area network (WBAN) is a sub-field of WSNs and it is the most emerging field to make a healthy world [2]. It is one of the wireless networks of communication between sensor devices of low power used in the human body and it is the compact communication medium via microphones, headphones, sensors, etc. The technology of WBAN is still in its preliminary phase but used for a variety of applications, including healthcare, consumption of electronic devices, entertainment, military, and others.

According to the standardization of IEEE 802.15.6 classification, WBAN applications are broadly categorized into two types such as:

1. Healthcare Applications: The healthcare applications are wearable health monitoring Asthma, Aiding Professional and Armature Sports Training, Sleeping Staging, Assessing Soldier Fatigue and Battle Readiness, Patient monitoring, Cardiovascular diseases, Tele-medicine Systems and Ambient Assisted Living (AAL).

2. Non-healthcare Applications: The Non-healthcare applications are Entertainment applications, Realtime streaming, Emotion detection, secure authentication, and Emergency services.

The main aim of WBAN is to provide quality of service and improve patient's health that allows the medical practitioners to respond in a timely manner from any remote location. In the modern era, it plays a vital role as on average every family spends almost $20 \%$ of their income on medical bills. In WBAN technology, sensor nodes are placed on the surface of the human body or embedded in the body to monitor vital signs like glucose monitoring, blood pressure, body temperature, and heart rates, etc. with the help of a wireless medium. As shown in Figure 1, the working steps of WBANs technology has three functional stages.

A. The first phase is known as the patient monitoring where all tiny sensor nodes are placed on the human body at different positions for continuous health monitoring. These nodes transmit sensed data to the sink node using single-hop and multi-hop routing topologies discussed [3].

B. In the second phase in known as the transmission and storage phase or central storage where all the gathered data from the patient monitoring system by sensor nodes are stored in the central server through proper Internet Server Provider (ISP).

In the final phase, medical practitioners analyze the gathered data of patient's data which are stored in the server and respond to patient's critical health from any remote location. It is also very cost-effective as it reduces the expenditure of patients by allowing patients to stay at their own house or place. 


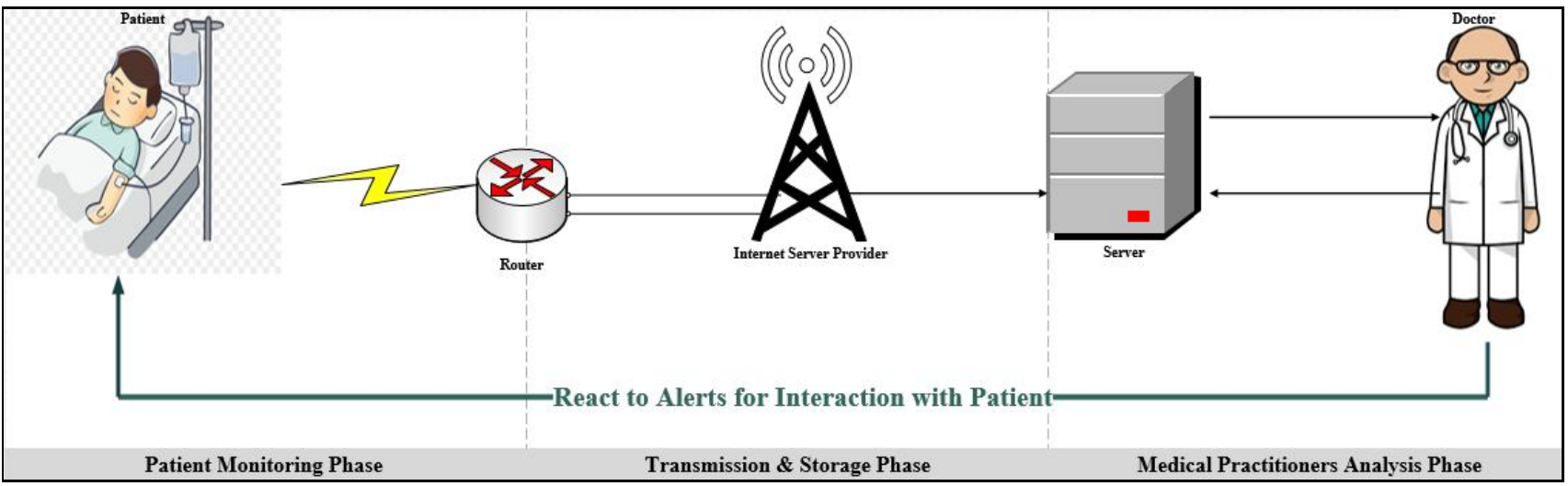

Figure 1: Working of WBAN Technology

In WBAN technology, tiny sensor nodes are typically powered with batteries and have limited energy or battery power resources. To extend the overall network lifetime of designed WBAN model, secure and efficient routing protocols play a vital role that can minimize energy consumption rates which are required to transmit the gathered data from sensor nodes to sink node. There are many routing protocols proposed for energy-efficient routing in WSN [4]-[5]. The concept of routing protocol which is used in WSN is not directly adaptable in WBAN as their individual working architectures. In WBAN technology, there are very few routing protocols for gathered data/packet transfer from patient to doctor or patient's guardian. Constrained Markov Decision Processes (CMDP) is one of them and it is proposed in [6] for intelligent WBAN model. In this model, data transmission between sensor nodes and sink nodes takes place in a multi-hop manner based on the concept of artificial intelligence. Each sensor node in this WBAN model transfers the sensed data to the nearest next-hop via the nearest relay, base station, sink node, etc.

The concept of data security with the energy-efficient approach is missing in these models, so we designed a model in which secure and energy-efficient routing of WBAN technology is used [7]. The architecture of the developed WBAN model id shown in Figure 2 and their explanation is written below.

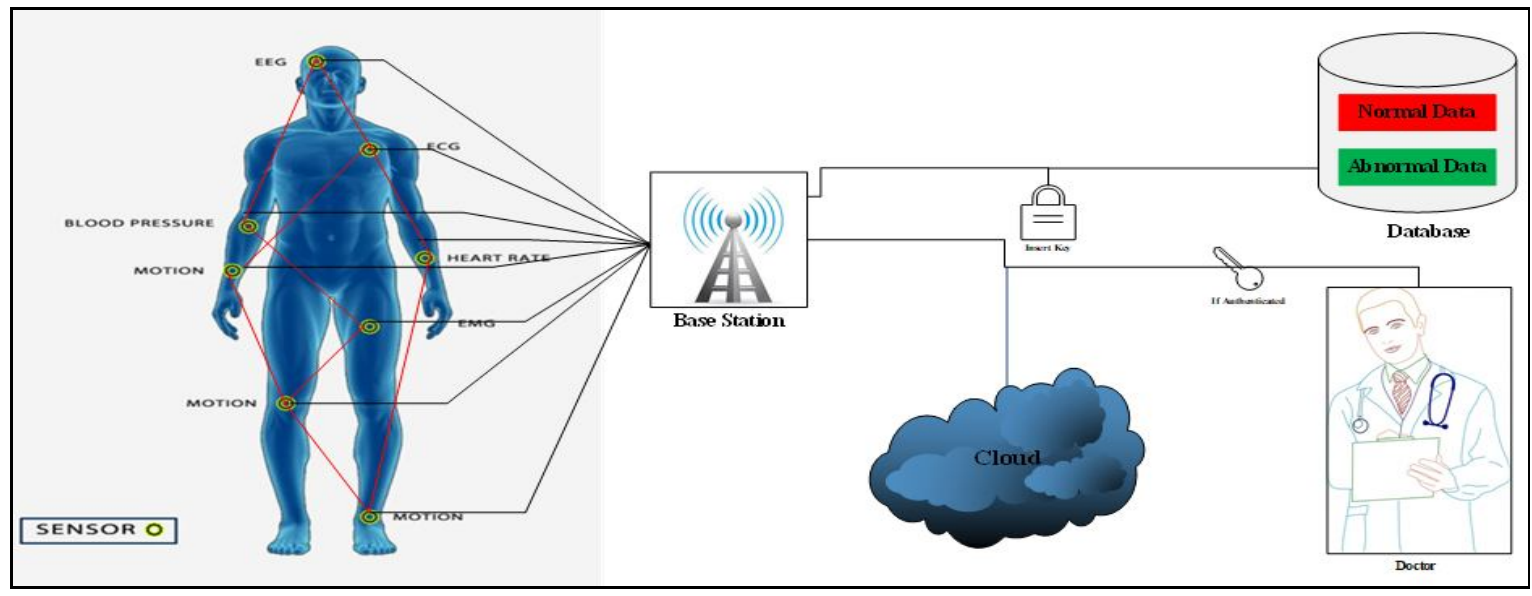

Figure 2: Architecture of Secure and Energy Efficient WBAN Technology

In this scenario, physiological data packets are generated at the sensor nodes in a constant rate and transmitted to the relay or central nodes using the concept of energy-efficient routing mechanism [8]. At the relay or central nodes, the sensed data packets are temporarily held in a queue waiting for the transmissions to the base station. At the same time, a data security control scheme is applied to authenticate patients or doctors. In this scenario, inter-WBAN, Intra-WBAN, and beyond-WBAN communication concepts are used to achieve maximum throughput with minimum transmission delay [9]. Wireless transmitting overhead is also reduced which leads to a noticeable reduction in energy consumption rate [10].
We demonstrate the efficiency of the proposed Intelligent Health Monitoring (IHM) System based on Secure Distributed Routing in the WBAN model through simulations. Besides, we compare designed IHM system with existing works based on the Quality of Service (QoS) parameters. The remaining research paper is organized as follows. In Sect. 2, related works are reviewed to analyze the secure or intelligent routing concept in WBAN. Sect. 3 describes the architecture of the IHM system. Sect. 4 presents the working of the proposed secure and energy-efficient routing protocol using the concept of artificial intelligence techniques. Simulation results and their comparison are presented in Sect. 5 based on the QoS parameters. Finally, Sect. 6 draws a conclusion and future scope of WBAN technology in the medical era. 
Rajeev Sharma et al., International Journal of Advanced Trends in Computer Science and Engineering, 9(4), July - August 2020,5138 - 5147

\section{RELATED WORK}

In this section, we review some of the state-of-art based on the secure routing protocols in WBAN technology using different techniques. W. Zang et al. in 2019 have introduced CMDP-based savvy transmission for remote body territory arrange in remote wellbeing checking. In this paper, they have contemplated the joint planning and affirmation control issue for WBAN-based remote wellbeing checking applications. By utilizing the obliged Markov choice procedures approach, an astute transmission calculation is proposed to together advance the vitality proficiency of door hub and WBAN sensor hub. Reproduction results are given to show that the proposed calculation fundamentally outflanks the insatiable plan (as far as throughput) and other planning plans which don't consider the intra WBAN connect (regarding power utilization for WBAN sensor hub). The conceivable point for future work is to apply a support learning approach that doesn't require any prior measurable information and consider increase heterogeneous WBAN sensor hubs. For this situation, the measure of framework states can be immense, in this manner basic learning ought to be examined and misused to diminish the calculation unpredictability and quicken the combination rate. The power utilization pace of the structured model is high for its perplexing engineering.

A. Samanta and S. Misra in 2018 have proposed vitality proficient and dispersed system the executives cost minimization in shrewd remote body territory systems. The proposed system endeavors to limit the dynamic network, obstruction of the board, and information scattering costs for astute WBAN. They have, hypothetically, broken down the presentation of the proposed system to give dependable information transmission in shrewd WBANs. Re-enactment results show a huge improvement in the system execution contrasted with the current arrangements yet they don't chip away at the evaluating instrument for sharp information spread in WBANs. So improvement required by utilizing the idea of better set up course for information transmission which is free from the human postural changes.

In 2010, Y. Ren et al. have introduced a model for observing patients through a protected and versatile medicinal services framework. In this work, the assorted variety of elements that participate in the plan of solid, clever, secure patient observing and the board frameworks has been displayed by creators and they depict the issues concerning body sensor systems (BSNs), demonstrating a few strategies that could be utilized to upgrade the usefulness of BSNs. Finally, they exhibit the procedures utilized by current secure m-human services frameworks to address patients' worries about security and protection within the sight of vindictive hubs and bargained situations. They utilized the idea of trust the board for secure information transmission however the system can just speak with intra model. In the event of between body correspondences, the system gets influenced, because of this administration deferral of the system, which is high and needs to limit by utilizing the streamlining calculations.

A. M. Rahmani et al. in 2018 have planned a framework for misusing shrewd e-Health portals at the edge of medicinal services Internet-of-Things (IoT) utilizing the idea of mist figuring and Internet-of-Things. Savvy passages at the closeness of sensor hubs in the keen home or clinic premises can abuse their novel key position to handle numerous difficulties in IoT-based wellbeing frameworks, for example, portability, vitality productivity, and adaptability, interoperability, and unwavering quality issues. The proposed full framework show incorporates every one of the information stream forms from information securing at sensor hubs to the cloud and end-clients. To keep up the proposals process, systems devour more vitality which isn't satisfactory in this advanced time.

In 2016, Qi Liu et al. presented a theoretical way to deal with spatial-fleeting effectiveness with multi-target advancement in a heterogeneous cloud condition. In this work, a versatile strategy is introduced by creators for going for spatial-fleeting proficiency in a heterogeneous cloud condition. An expectation model dependent on an advanced Kernel-based Extreme Learning Machine calculation is proposed for a quicker estimate of employment execution length and space occupation, which thusly encourages the procedure of undertaking planning through a multi-target calculation called existence improved NSGA-II (TS-NSGAII). From the test consequences of this work, the proposed methodology can spare surmised 47-55s averagely on each undertaking execution. Inferable from the absence of streamlining approaches, the exhibition of the technique is low.

A. Khalid et al. in 2018 have exhibited a model of towards dynamic coordination among home machines utilizing multitarget vitality streamlining for interest side administration in savvy structures. The proposed strategy finds the most ideal timetable of each home machine thinking about framework limitations. The planning is done dependent on day-ahead and constant premise. They didn't build up any relationship of the power cost, PAR, and holding up time to upgrade the exhibition of the framework. To make the booking dynamic, they had intended to fuse the idea of coordination among the homes to augment the client comfort and to limit the power cost.

Multi-sensor combination in WBAN is proposed by the $\mathrm{R}$. Gravina et al. in 2017 and they examined clear inspirations and points of interest of multi-sensor information combination and especially cantered on physical action acknowledgment, targeting giving an orderly classification and basic correlation structure of the writing, by recognizing unmistakable properties and parameters influencing information combination plan decisions at various levels (information, highlight, and choice). They additionally spread information combination in the spaces of feeling acknowledgment and general-wellbeing and present applicable bearings and difficulties of future research on multi-sensor combination in the BSN area.

After studying existing research work for routing in the WBANs, the following drawbacks are identified:

* CMDP based approach in the WBAN technique having the highest energy consumption rate and it is also unsecured due to lack of security constraint.

* Existing work is merely limited to intra-body communication in WBANs environment, which inherently increases the energy consumption rate of the network.

* For the assortment of cluster heads/relays nodes in existing work, the concept of minimal distance from the base station or sink node is used. But the concept of the node with the highest residual energy is not used, so the data 
Rajeev Sharma et al., International Journal of Advanced Trends in Computer Science and Engineering, 9(4), July - August 2020,5138 - 5147

drop rate is high regarding the searching of next intermediated nodes.

Motivation and contributions: WBANs is a structured and heterogeneous network where the body sensor nodes have some residual energy which is power by external sources. The most important goal of any WBANs is to transmit the data from one sensor node to another sensor node within a network. The data packets transportation process requires a secure route discovery process due to which a location monitor is required in the network. As all the body sensor nodes have residual energy, then it is hard to point out at any particular sensor node which can act as a relay sensor node. So the selection of a relay sensor node is the biggest task in WBANS. From these types of challenging tasks, we present the IHM system based on secure distributed routing in the WBAN model through simulations. In simple words, this paper makes the following contributions to achieve better network performance.

* We present a novel routing algorithm based on the concept of clustering mechanism for successful packet data transmission and it is known as Secure Distributed and Energy Efficient Routing (SDEER) Protocol.
* For the selection of intermediate nodes or cluster heads/relays, Feed Forward Back Propagation Neural Network (F2-BPNN) algorithm is used with Particle Swarm Optimization (PSO) for differentiating the properties of the node.

* For the validation of the proposed IHM system, we evaluate QoS performance parameters like Throughput, Energy Consumption, and Queue Size with state-of-theart approaches.

This paper presents the IHM system based on the secure and energy-efficient routing mechanism using a hybrid approach by F2-BPNN with the PSO algorithm and their comparison with W. Zang et al. in 2019.

\section{IHM ARCHITECTURE}

The proposed Intelligent Health Monitoring (IHM) System based on a secure distributed and energy-efficient routing mechanism in WBAN technology using the F2-BPNN with the PSO algorithm. The block diagram of the proposed IHM system shown in Figure 3.

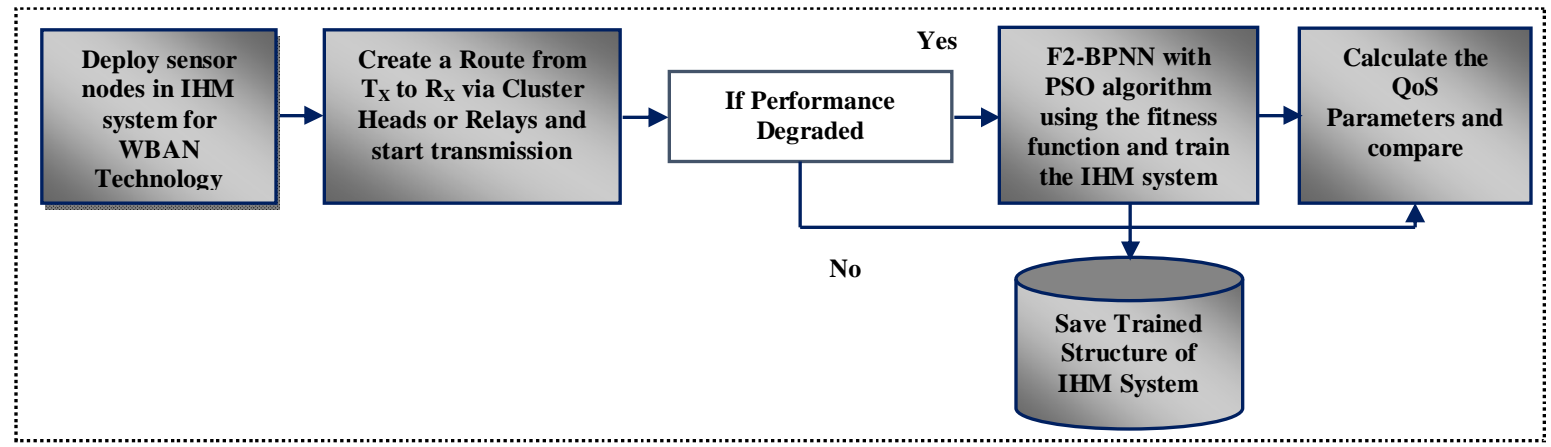

Figure 3: IHM System Block Diagram

The proposed IHM system mainly consists of the following four steps:

- IHM System Setup: This is the basic and initial step for the designing of a WBAN model. In this step, sensor nodes are deployed and a wireless connection is established among all other sensor nodes, and in section 3.1 , the detailed explanation of this step is described with the algorithms.

Table 1: Symbol Description

\begin{tabular}{|c||l|}
\hline \multicolumn{1}{|c|}{ Symbol } & \multicolumn{1}{|c|}{ Description } \\
\hline $\mathbf{N}$ & Sensor Nodes \\
\hline $\mathbf{N}_{\mathbf{P}}$ & Sensor Nodes Properties \\
\hline $\mathbf{A}_{\mathbf{R}}$ & Area of IHM Simulator \\
\hline $\mathbf{W}$ & Width of IHM Simulator \\
\hline $\mathbf{H}$ & Height of IHM Simulator \\
\hline $\mathbf{T}_{\mathbf{X}}$ & Transmitter or source sensor node \\
\hline $\mathbf{R}_{\mathbf{X}}$ & A receiver or destination node \\
\hline $\mathbf{C H}_{\mathbf{H}}$ & Cluster head \\
\hline $\mathbf{E}_{\mathbf{R}}$ & The residual energy of sensor nodes \\
\hline $\mathbf{E T}_{\mathbf{X}}$ & $\begin{array}{l}\text { The energy consumed by nodes during } \\
\text { sensed data packets transmission }\end{array}$ \\
\hline $\mathbf{E R}_{\mathbf{X}}$ & $\begin{array}{l}\text { The energy consumed by nodes during } \\
\text { receiving of data packets }\end{array}$ \\
\hline $\mathbf{E}_{\mathbf{A M P}}$ & Amplification energy of nodes \\
\hline
\end{tabular}

\begin{tabular}{|c|l|}
\hline $\mathbf{P}_{\text {DATA }}$ & Packet data sensed by sensor nodes \\
\hline $\mathbf{R a n d}$ & A random number between $0 \rightarrow 1$ \\
\hline $\mathbf{S}_{\mathbf{N}}$ & Sink Node \\
\hline $\mathbf{D F F}$ & Destination Found Flag \\
\hline $\mathbf{R}_{\mathbf{T}}$ & Route from $\mathrm{T}_{\mathrm{X}}$ to $\mathrm{R}_{\mathrm{X}}$ \\
\hline $\mathbf{O R}_{\mathbf{T}}$ & Optimized Route from $\mathrm{T}_{\mathrm{X}}$ to $\mathrm{R}_{\mathrm{X}}$ \\
\hline $\mathbf{C}_{\mathbf{L I M I T}}$ & Coverage limit $=25 \%$ of $\mathrm{A}_{\mathrm{R}}$ \\
\hline $\mathbf{C}_{\mathbf{S E T}}$ & Coverage set of sensor nodes \\
\hline \hline
\end{tabular}

- Route Discovery: In this step, we use the concept of clustering approach to select a relay or cluster head node sometimes also referred to as an intermediate/forwarder node which plays a vital role in sensed data transmission by sensor node in the human body. The Discovery of the route is the bottleneck step of our proposed routing protocol in the IHM system which is described in Sect. 3.2 with algorithmic steps.

- Route Optimization: After the route discovery, sensed data to be transferred from the $T_{X}$ to $R_{X}$ with minimum energy consumption rate, but discovered route not transfer the data packet accurately then we need to optimize the route. Route optimization is performed by using the concept of hybridization of F2-BPNN with PSO as an optimization technique and the route optimization process is described in Sect. 3.3. 
Rajeev Sharma et al., International Journal of Advanced Trends in Computer Science and Engineering, 9(4), July - August 2020,5138 - 5147

- Performance Evaluation: This step is used to validate the performance of the IHM system by comparing the state of arts and description is given in the Sect. 3.4.

\subsection{IHM System Setup}

The system setup of the proposed IHM system based on a secure and energy-efficient routing mechanism for WBAN communication using consists of several steps. The procedures of the IHM system are defined as follows:

A. IHM Framework: Firstly we design and develop an HIM framework using the concept of Graphical User Interface (GUI) for simulation of the proposed WBAN model. The area of simulation for the proposed IHM system is defined by using given equation 1 .

$$
\text { IHM - FrameworkArea, } A=H \times W
$$

Where $\mathrm{H} \leftarrow$ Height of IHM System

$\mathrm{W} \leftarrow$ Width of IHM System and both are considered as $1000 \mathrm{~m}$ (a meter is just used for scaling), so the total area becomes $1000 \mathrm{~m}^{2}$.The designed framework is shown in Figure 4.

B. Sensor Node Placement: After the development of IHM framework, $\mathrm{N}$ sensor node is deployed in this framework for simulation and defined $\mathrm{T}_{\mathrm{X}}$ and $\mathrm{R}_{\mathrm{X}}$ sensor node which is shown in the Figure 4 with their algorithm written as:

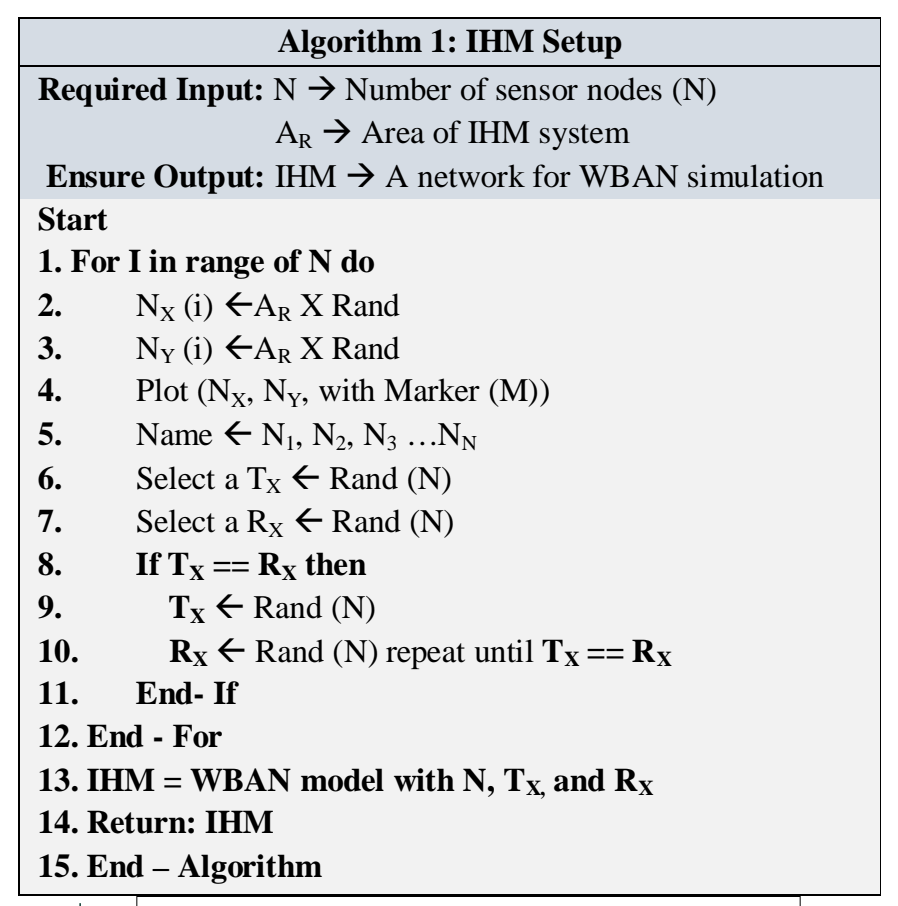

In this framework, we consider some assumptions for the development of the IHM system using the area of the simulator. The assumptions are written as:

$\checkmark \quad$ All deployed sensor nodes nature are stationary

$\checkmark \quad$ Sink node and other nodes like cluster head or relay nodes have equal capabilities

$\checkmark \quad$ The position of sink node is the center of the human body with unlimited resources of energy and is responsible for computing received data and helps to create a route from the $\mathrm{T}_{\mathrm{X}}$ to $\mathrm{R}_{\mathrm{X}}$ in the WBAN model.

$\checkmark$ All deployed sensor nodes in the body initially have equal energy for simulation and all sensor nodes transmit data to sink node using the concept of single as well as multi-hop routing topology.

C. Sensor Nodes Coverage Area Calculation: Set the communication range (25\% of the entire area according to the IEEE standard) then define the coverage area for each sensor nodes using the written algorithm:

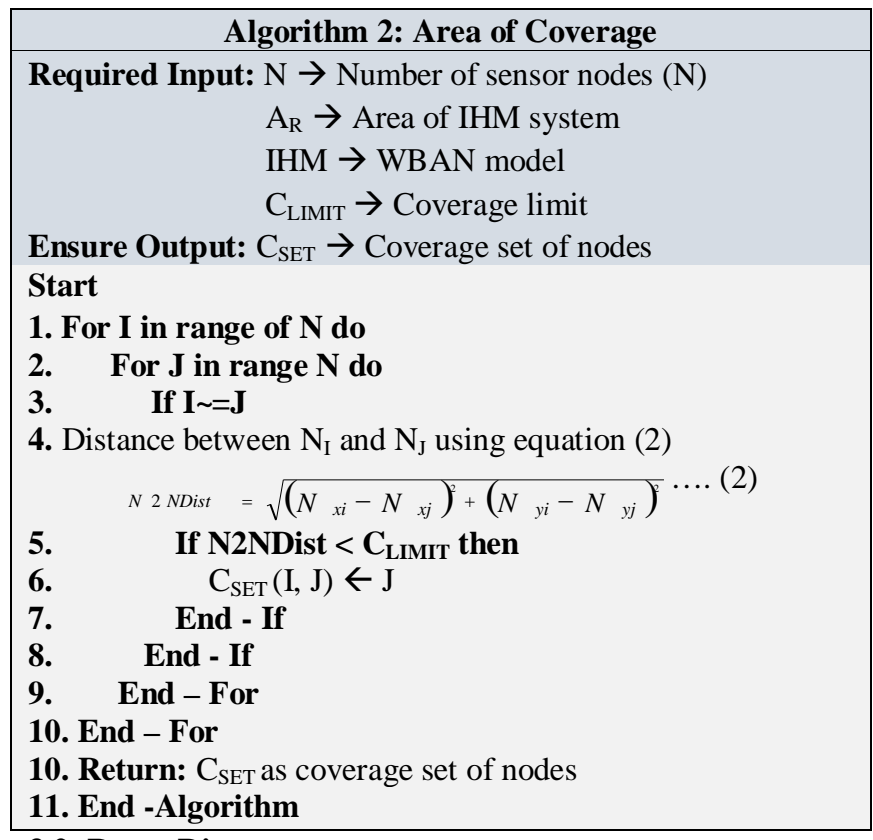

3.2 Route Discovery

Sensed data packet by sensor nodes is transmitted using the route and in the IHM system, SDEER protocol is used for route discovery from $T_{X}$ to $R_{X}$ and then calculate the QoS parameters of WBAN model. The algorithm of SDEER protocol is written as:

\begin{tabular}{l} 
Algorithm 3: SDEER Protocol \\
\hline Required Input: $\mathrm{T}_{\mathrm{X}} \rightarrow$ Source or Transmitter Node \\
$\qquad \mathrm{R}_{\mathrm{X}} \rightarrow$ Destination or Receiver Node \\
$\qquad \mathrm{C}_{\mathrm{SET}} \rightarrow$ Coverage set of Nodes \\
$\qquad \mathrm{E}_{\mathrm{R}} \rightarrow$ Residual Energy of Nodes \\
Ensure Output: $\mathrm{R}_{\mathrm{T}} \rightarrow$ Route from $\mathrm{T}_{\mathrm{X}}$ to $\mathrm{R}_{\mathrm{X}}$ \\
Start \\
1. Initialize the WBAN parameters \\
2. Deployment of the Sink Node $\left(\mathrm{S}_{\mathrm{N}}\right)$ in the WBAN \\
3. Calculate distance of $\mathrm{N}$ form $\mathrm{S}_{\mathrm{N}}, \mathrm{N}-\mathrm{S}_{\mathrm{N}} \mathrm{DIST}$ \\
4. For I in range of $\mathrm{N}$ do \\
5. If $\mathrm{E}_{\mathbf{R}}\left(\mathrm{N}(\mathrm{I})\right.$ ) is maximum and $\mathrm{N}-\mathrm{S}_{\mathrm{N}}$ DIST is distributed \\
equally
\end{tabular}




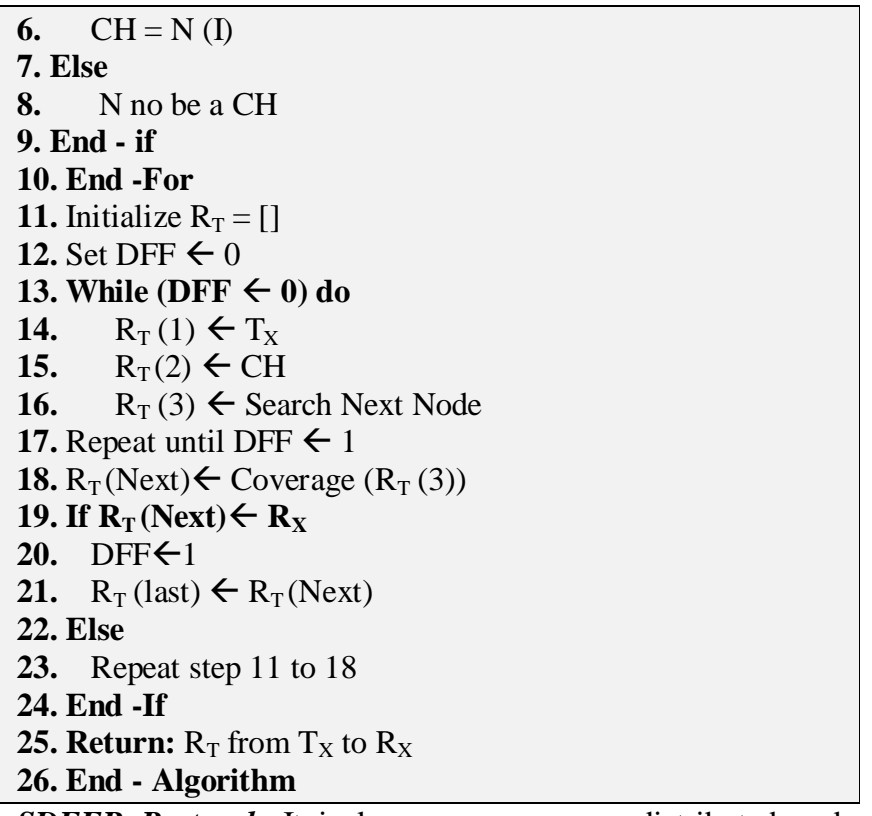

SDEER Protocol: It is known as a secure distributed and energy-efficient routing protocol which helps the IHM system for communication purpose between $T_{X}$ and $R_{X}$ via cluster heads/relays. This routing protocol works based on the clustering mechanism using the concept of the residual energy of the sensor but not consider the security phenomenon. After the data transmission using the discovered route, we calculate the QoS parameters of the IHM model and if the performance not up to acceptance, then we need to verify the status of the node and create a new route using the combination of F2BPNN with PSO which process is known as the route optimization.

\subsection{Route Optimization}

It is the most effective step of the proposed IHM system to detect and prevent the model from the attackers/malicious/fail nodes. In this mechanism, firstly PSO is used to optimize the sensor nodes properties according to the fitness function. If any nodes satisfy the fitness criteria, then considered as normal communicating nodes otherwise consider as the suspected sensor nodes. Route optimization process consists of two different approaches mentioned below:

A. Nodes Properties Optimization: When the IHM system is created, some basic parameters are initially provided to each sensor nodes like coordinates $(\mathrm{X}, \mathrm{Y})$, residual energy $\left(E_{R}\right)$, Transmission rate, delay, drop rate, etc. Based on these properties, SDEER protocol with F2BPNN can't identify the appropriate intermediate nodes when multi-hop routing is performed. Where F2BPNN is used as a classifier to classify the suspected or normal communicating nodes and its work based on their training. If the properties of the node are unique and appropriate, then the network train properly otherwise network performance affected.

B. Malicious/Fail Node Detection: In this step, suspected nodes are classified as malicious/fail/attacker nodes or normal communicating nodes based on their optimized properties. To complete this action in the IHM system, F2BPNN is used as the classifier.
Route optimization is needed when the network performance of the network is degraded, otherwise no need to optimize the route. To optimize the route, SDEER is used with the combination of F2BPNN with PSO, and their algorithm is written as:

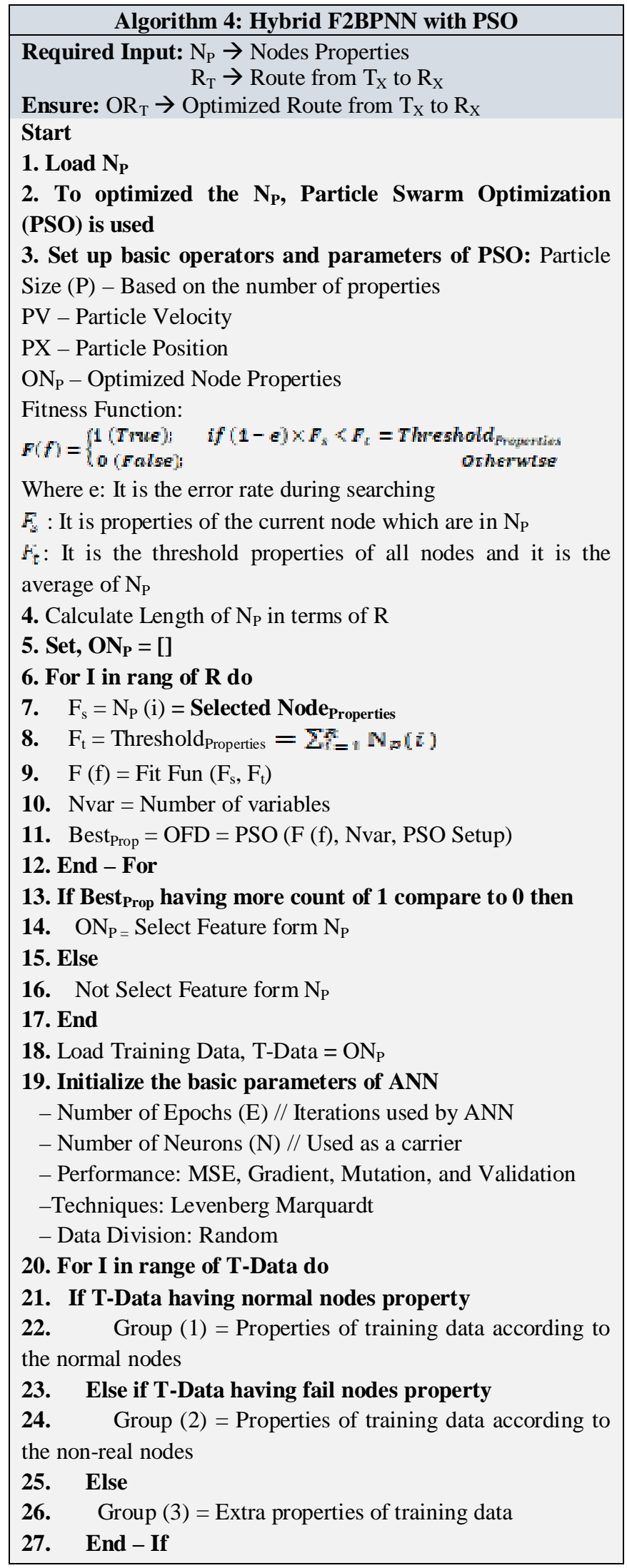




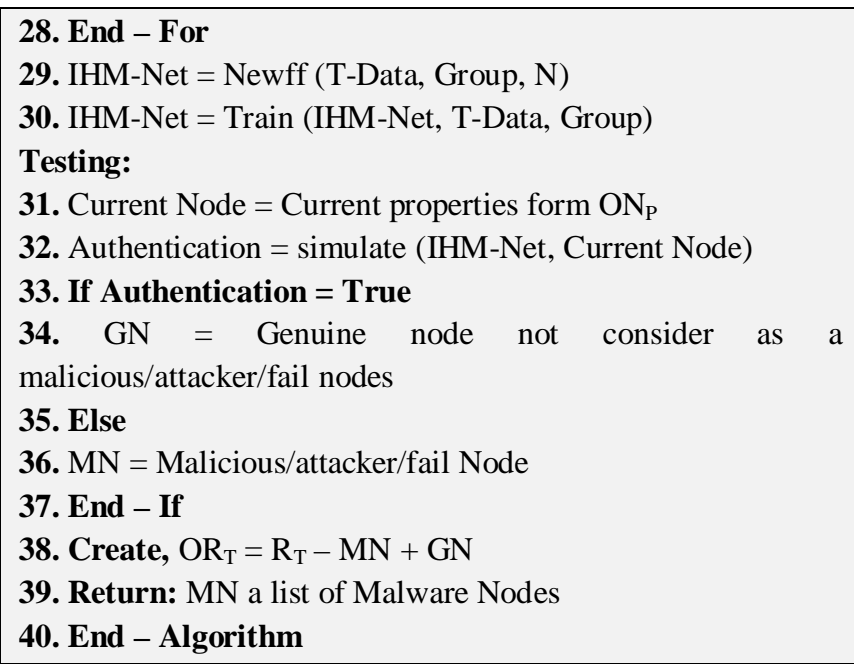

3.3. Performance Evaluation

The HIM system performance evaluation is one of the essential steps to validate the proposed scenario by using the combination of F2BPNN with PSO based SDEER protocol. To validate the proposed IHM model, we compare the evaluated parameters such as Throughput, Energy Consumption, and Queue Size using SDEER protocol and SDEER protocol with PSO based F2BPNN and also compare with the existing state of arts. The used QoS parameters are described below.

A. Throughput: Throughput is the number of messages/data successfully delivered per unit time.

$$
\text { Throughput }=\frac{\sum_{i=1}^{N} \text { PDATA } \text { Delivered }_{\text {Sentime }} \times \text { PDATA }}{\text { Averagesiz }} \text { PDATA ...... (3) }
$$

B. Energy Consumption: It is the specified summation of how much energy consumed by a node for a bit of data to transmission across the WBAN model from $T_{X}$ to $R_{X}$.

$$
E_{\text {Consumption }}=\sum_{i=1}^{\text {Node }} E_{R}-E_{T X}+E_{R X}+E_{A M P} \ldots
$$

Queue Size: It is the count of all data packets which are in the queue for the transmission from the $T_{X}$ to $R_{X}$ in a unit second.

\section{IHM SYSTEM DESCRIPTION}

The major challenge of this research work is to detect classify the malicious/fail/attacker nodes based on their properties using F2BPNN with the PSO algorithm as a route optimization technique. To achieve this target, we introduce a secure distributed energy-efficient transmission for interdomain, intra-domain, and beyond-domain. We establish the three-domain architecture for routing via cluster head, sink, and intermediate nodes and we proposed effective distance as well as residual energy-based searching approach for the optimal transmission. The simulation model of the proposed IHM system is shown in Figure 5.

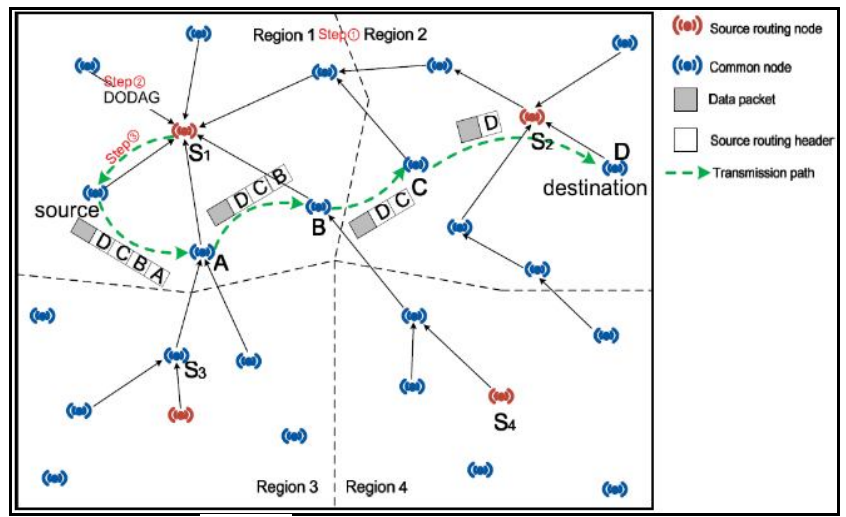

Figure 5: IHM Simulation Model

The above Figure 5 represents the IHM simulator with an area of $1000 \mathrm{~m}^{2}$ where the total area is divided into four subregions and in each region, one node is selected as cluster head and the data packet is transmitted in a sequence. The discovered rote is represented by the green color dotted line which is known as the transmission path. For the simulation of the IHM system, we consider some basic requirements which are given in table 2 .

Table 2: IHM System Parameters

\begin{tabular}{|l|l|}
\hline \multicolumn{1}{|c|}{ Parameters } & \multicolumn{2}{c|}{ Description } \\
\hline Nodes & 5-50 \\
\hline Placement & Randomly with Area \\
\hline Area & 1000m ${ }^{2}$ \\
\hline Simulation Tool & MATLAB \& SIMULINK \\
\hline Clusters & Based on SDEER Protocol \\
\hline Routing Protocol & OANN-EATSRA \\
\hline Packets Size & 5000 bits \\
\hline Optimizer & PSO \\
\hline Classifier & F2BPNN \\
\hline $\begin{array}{l}\text { Authentication } \\
\text { Parameter }\end{array}$ & $\begin{array}{l}\text { Energy Consumption and } \\
\text { Throughput }\end{array}$ \\
\hline $\begin{array}{l}\text { Evaluation } \\
\text { Parameter }\end{array}$ & $\begin{array}{l}\text { Throughput, Energy } \\
\text { Consumption, and Queue Size }\end{array}$ \\
\hline
\end{tabular}

Based on the above-mentioned parameters and simulation model, we simulate the model and validate the developed model based on the QoS parameters in the Sect 5, and for the simulation, we use MATLAB \& SIMULINK software with the optimization and artificial intelligence toolboxes.

\section{RESULTS AND DISCUSSION}

We have performed simulations of the IHM system to show the efficiency of our proposed SDEER protocol using the F2BPNN with PSO. We have also compared the results of the proposed IHM system with the CMDP technique developed by $W$. Zang et al. in 2019. We have implemented all the algorithm and protocols using the MATLAB R2016a software and executed on Windows OS (Operating System). Based on the above-mentioned scenario in table 2, the simulation results of the proposed IHM system with $W$. Zang et al. work are given as: 
Table 3: Throughput Comparison

\begin{tabular}{|c||c|c|}
\hline $\begin{array}{c}\text { A verage Energy } \\
\text { Consumption }\end{array}$ & W. Zang et al. & $\begin{array}{c}\text { IHM } \\
\text { System }\end{array}$ \\
\hline $\mathbf{2 0}$ & 0.7933 & 1.937 \\
\hline $\mathbf{2 5}$ & 0.8364 & 2.1933 \\
\hline $\mathbf{3 0}$ & 1.1843 & 2.4844 \\
\hline $\mathbf{3 5}$ & 1.2452 & 2.9233 \\
\hline $\mathbf{4 0}$ & 1.3844 & 3.0644 \\
\hline $\mathbf{4 5}$ & 1.4939 & 3.2493 \\
\hline $\mathbf{5 0}$ & 1.5834 & 3.5322 \\
\hline $\mathbf{5 5}$ & 1.6389 & 3.9742 \\
\hline $\mathbf{6 0}$ & 1.8374 & 4.1293 \\
\hline \hline
\end{tabular}

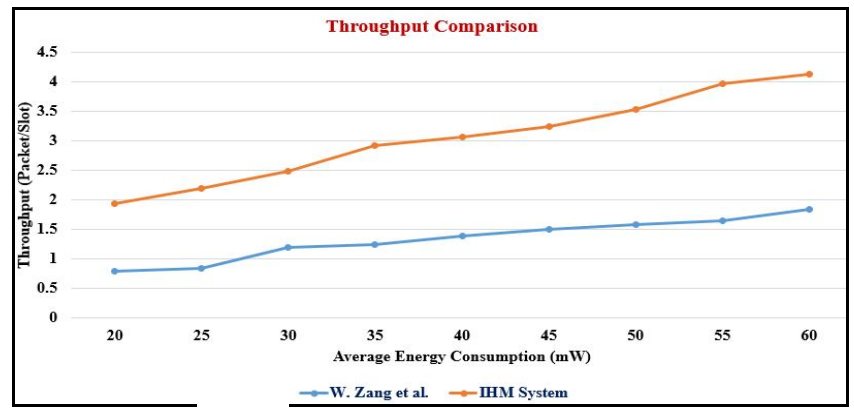

Figure 6: Throughput Comparison

The throughput of the proposed IHM system is compared with the W. Zang et al. work in Figure. 6 and we observed the throughput of the IHM system is improved. This improvisation is done using the SDEER routing protocol based on the F2BPNN with the PSO algorithm. The number of sensed data packets received by the sink node is known as the throughput and it should be maximum. The throughput of the WBAN model depends on the number of sensor nodes that are successfully participating or active in a WBAN. The number of data packets sent by $\mathrm{T}_{\mathrm{X}}$ node to the sink node is directly proportional to the number of successfully participating active or alive sensor nodes in the network. Especially in the case of the WBAN model, the sensed patient data packets are important and crucial and need a secure route for transmission. So, it is rattling significant to minimize the data packet loss in the IHM system and also maximize the throughput. As shown in Figure 6, our proposed SDEER protocol has more throughput as compared to CMDP protocol by using the concept of hybridization of F2BPNN with the PSO algorithm. Other QoS parameters are described below section of this paper and all are improved as compared to the existing work.

Table 4: Energy Consumption Comparison

\begin{tabular}{|c||c|c|}
\hline $\begin{array}{c}\text { Average Energy } \\
\text { Consumption }\end{array}$ & \multicolumn{1}{c|}{ W. Zang et al. } & $\begin{array}{c}\text { IHM } \\
\text { System }\end{array}$ \\
\hline $\mathbf{2 0}$ & 1.244 & 0.736 \\
\hline $\mathbf{2 5}$ & 1.573 & 0.937 \\
\hline $\mathbf{3 0}$ & 1.937 & 1.348 \\
\hline $\mathbf{3 5}$ & 2.384 & 1.493 \\
\hline $\mathbf{4 0}$ & 2.891 & 1.938 \\
\hline $\mathbf{4 5}$ & 3.683 & 2.364 \\
\hline $\mathbf{5 0}$ & 3.927 & 2.874 \\
\hline $\mathbf{5 5}$ & 4.738 & 2.981 \\
\hline $\mathbf{6 0}$ & 5.184 & 3.746 \\
\hline \hline
\end{tabular}

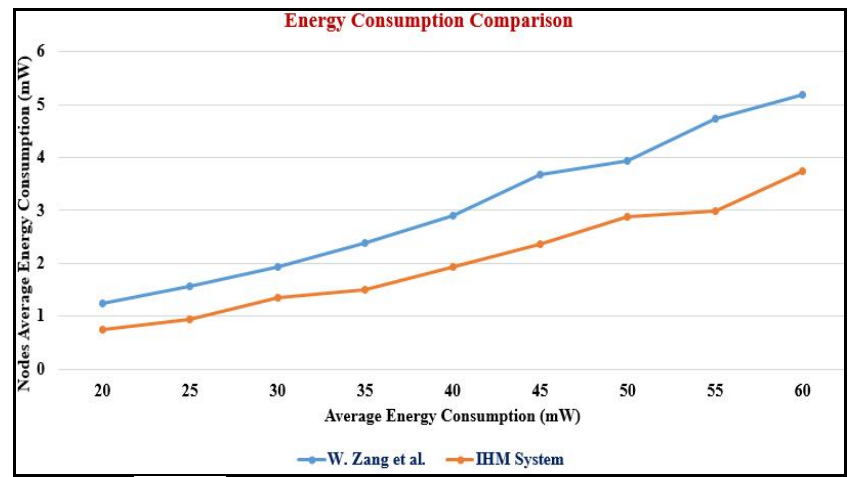

Figure 7: Energy Consumption Comparison

In this experiment, we have considered average energy consumption as a QoS performance measure and compared SDEER protocol with CMDP protocol by $W$. Zang et al. It is very important to maintain higher residual energy of sensor nodes to prolong the IHM network lifetime. We can see from Figure 6, that average energy consumption of the IHM system is less than CMDP protocol and it is possible by utilizing the concept of SDEER routing protocol based on the F2BPNN with the PSO algorithm. We have achieved these results by employing SDEER routing topology in WBAN models which work based on the clustering approach based on the sensor node residual energy.

Table 5: Queue Size Comparison

\begin{tabular}{|c|c|c|}
$\begin{array}{c}\text { A verage Energy } \\
\text { Consumption }\end{array}$ & W. Zang et al. & $\begin{array}{c}\text { IHM } \\
\text { System }\end{array}$ \\
\hline \hline $\mathbf{2 0}$ & 5.81 & 3.84 \\
\hline $\mathbf{2 5}$ & 5.85 & 3.86 \\
\hline \hline $\mathbf{3 0}$ & 5.87 & 3.89 \\
\hline $\mathbf{3 5}$ & 5.71 & 3.22 \\
\hline $\mathbf{4 0}$ & 5.79 & 3.41 \\
\hline $\mathbf{4 5}$ & 5.8 & 3.82 \\
\hline $\mathbf{5 0}$ & 5.81 & 3.85 \\
\hline $\mathbf{5 5}$ & 5.78 & 3.66 \\
\hline $\mathbf{6 0}$ & 5.72 & 3.28 \\
\hline \hline
\end{tabular}

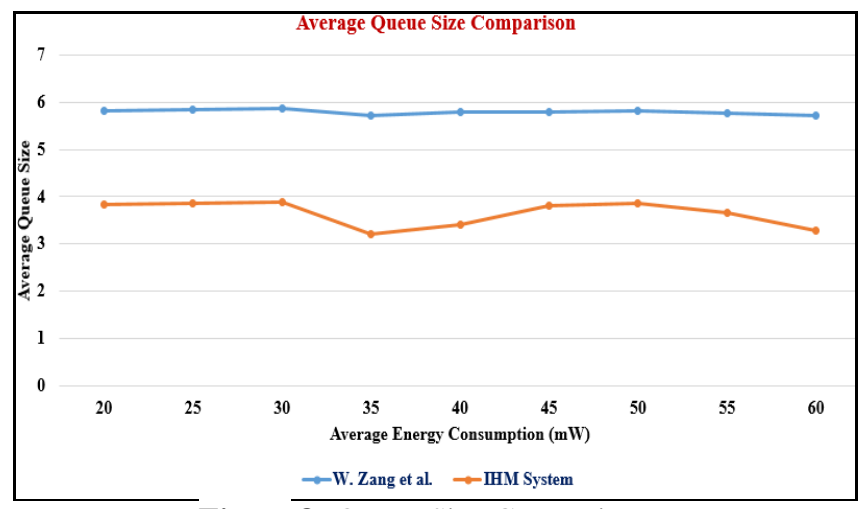

Figure 8: Queue Size Comparison

In the simulation of the IHM system, we have evaluated the efficiency of the proposed SDEER protocol in terms of queue size. The queue size parameters in the WBAN technology are used to identify how much data packets are feeling traffic during the transmission from the $T_{X}$ to $R_{X}$ node based on the nodes' residual energy and distance to the sink 
node. Overall we founded that, the impact of SDEER protocol based on the F2BPNN with the PSO algorithm has achieved a noticeable result in the era of WBAN technology.

\section{CONCLUSION AND FUTURE WORK}

In this paper, an IHM system based on the SDEER protocol in WBAN technology is proposed using the concept of the F2BPNN with the PSO algorithm. WBAN technology in the IHM system is highly significant as continuous monitoring of patients' health is done in real-time. IHM system also facilitates medical practitioners to observe critical health conditions of patients remotely from anywhere or any location. However, the usage of IHM systems in real-time is very limited as maintaining the limited residual energy of the sensor nodes is very difficult. So, in this research paper, the SDEER protocol based on the F2BPNN with the PSO algorithm is implemented as an optimized single as well as multi-hop routing with the clustering mechanism. We have considered a feed-forward approach with a back propagation technique to select cluster heads or relays or intermediate sensor nodes based on their residual energy and distance from the $\mathrm{R}_{\mathrm{X}}$ or sink node. We have performed simulations of the IHM system to evaluate the efficiency of our proposed scheme and also compared it with state-of-art protocols like CMDP. The simulation results are clearly shown that our proposed IHM system is significantly prolonged the lifetime of the network and reduce the energy consumption rate.

In future work, CNN will be used as a classifier to train system based on hybridization of another classifier or swarm-based optimization technique which may help to reduce the transmission time of the IHM system with minimum energy consumption.

\section{REFERENCES}

[1] Chavva, S. R., \& Sangam, R. S. (2019). An energyefficient multi-hop routing protocol for health monitoring in wireless body area networks. Network Modeling Analysis in Health Informatics and Bioinformatics, 8(1), 21. https://doi.org/10.1007/s13721-019-0201-9

[2] Zang, W., Miao, F., Gravina, R., Sun, F., Fortino, G., \& Li, Y. (2019). CMDP-based intelligent transmission for wireless body area network in remote health monitoring. Neural computing and applications, 32(3), 829-837.

[3] Ahmad, A., Javaid, N., Qasim, U., Ishfaq, M., Khan, Z. A., \& Alghamdi, T. A. (2014). RE-ATTEMPT: a new energy-efficient routing protocol for wireless body area sensor networks. International Journal of Distributed Sensor Networks, 10(4), 464010.

[4] Afsana, F., Asif-Ur-Rahman, M., Ahmed, M. R., Mahmud, M., \& Kaiser, M. S. (2018). An energy conserving routing scheme for wireless body sensor nanonetwork communication. IEEE Access, 6, 91869200.

[5] Monowar, M. M., \& Bajaber, F. (2017). Towards differentiated rate control for congestion and hotspot avoidance in implantable wireless body area networks. IEEE Access, 5, 10209-10221.

[6] Yang, X., Wang, L., \& Zhang, Z. (2018). Wireless body area networks MAC protocol for energy efficiency and extending lifetime. IEEE sensors letters, 2(1), 1-4.
[7] Zang, W., Miao, F., Gravina, R., Sun, F., Fortino, G., \& Li, Y. (2019). CMDP-based intelligent transmission for wireless body area network in remote health monitoring. Neural computing and applications, 1-9.

[8] Samanta, Amit, and SudipMisra. Energy-efficient and distributed network management cost minimization in opportunistic wireless body area networks. IEEE Transactions on Mobile Computing 17.2 (2018): 376-389.

[9] Ren, Yonglin, Richard Werner, NelemPazzi, and AzzedineBoukerche. Monitoring patients via a secure and mobile healthcare system. IEEE Wireless Communications 17, no. 1 (2010): 59-65.

[10] Rahmani, Amir M., Tuan Nguyen Gia, BehailuNegash, Arman Anzanpour, ImanAzimi, Mingzhe Jiang, and PasiLiljeberg. Exploiting smart e-Health gateways at the edge of healthcare Internet-of-Things: A fog computing approach. Future Generation Computer Systems 78 (2018): 641-658. https://doi.org/10.1016/j.future.2017.02.014

[11] Liu, Qi, et al. A speculative approach to spatial $\square$ temporal efficiency with multi $\square$ objective optimization in a heterogeneous cloud environment. Security and Communication Networks9.17 (2016): 4002 4012.

[12] Khalid, Adia, Nadeem Javaid, Mohsen Guizani, MusaedAlhussein, Khursheed Aurangzeb, and Manzoorllahi. Towards dynamic coordination among home appliances using multi-objective energy optimization for demand side management in smart buildings. Ieee Access 6 (2018): 19509-19529.

[13] Gravina, Raffaele, ParastooAlinia, Hassan Ghasemzadeh, and Giancarlo Fortino. Multi-sensor fusion in body sensor networks: State-of-the-art and research challenges. Information Fusion 35 (2017): 68-80.

[14] Iqbal, Muhammad, Muhammad Naeem, AlaganAnpalagan, Ashfaq Ahmed, and Muhammad Azam. Wireless sensor network optimization: Multiobjective paradigm. Sensors 15, no. 7 (2015): 1757217620.

[15] Klemm, Maciej, and Gerhard Troester. Textile UWB antennas for wireless body area networks. IEEE Transactions on Antennas and Propagation 54, no. 11 (2006): 3192-3197.

[16] Tseng, Hsueh-Wen, Ruei-Yu Wu, and Yi-Zhang Wu. An efficient cross-layer reliable retransmission scheme for the human body shadowing in ieee 802.15. 6-based wireless body area networks. IEEE Sensors Journal 16, no. 9 (2016): 3282-3292.

[17]Zhu, Xiao-Qi, Yong-Xin Guo, and Wen Wu. A compact dual-band antenna for wireless body-area network applications. IEEE Antennas and Wireless Propagation Letters 15 (2016): 98-101. https://doi.org/10.1109/LAWP.2015.2431822

[18] Jermana M, Matheus R, Glauber V et al (2018) Advances in photopletysmography signal analysis for biomedical applications. Sensors 18(6): 1894

[19] Benharref A, Serhani MA (2014) Novel cloud and SOAbased framework for E-health monitoring using wireless biosensors. IEEE J Biomed Health Inf 18(1):4655

[20] Junnila S, Kailanto H, Merilahti J et al (2010) Wireless, multipurpose in-home health monitoring platform: two case trials. IEEE Trans Inf Technol Biomed 14(2):447-455 
Rajeev Sharma et al., International Journal of Advanced Trends in Computer Science and Engineering, 9(4), July - August 2020,5138 - 5147

[21] De Oliveira Júnior WG, Oliveira JMD, Munoz R et al. (2018) a proposal for internet of smart home things based on BCI system to aid patients with amyotrophic lateral sclerosis. Neural Comput Appl 2018:1-11

[22] Cavallari R, Martelli F, Rosini R et al (2014) A survey on wireless body area networks: technologies and design challenges. IEEE Commun Surv Tutor 16(3):1635-1657

[23] Patel M, Wang J (2010) Applications, challenges, and prospective in emerging body area networking technologies. IEEE Wirel Commun 17(1):80-88

[24] He C, Fan X, Li Y (2013) Toward ubiquitous healthcare services with a novel efficient cloud platform. IEEE Trans Biomed Eng 60(1):230-234

https://doi.org/10.1109/TBME.2012.2222404
[25] Mahmoud MME, Rodrigues JJPC, Ahmed SH et al (2018) Enabling technologies on cloud of things for smart healthcare. IEEE Access 2018:1

[26] Rodrigues JJPC, Segundo DBDR, Junqueira HA et al (2018) Enabling technologies for the internet of health things. IEEE Access 6:13129-13141

[27] Sharma R, Kang S. Challenges and Applications of Wireless Body Area Networks. International Journal of Innovative Technology and Exploring Engineering, Volume-8, Issue-9S (2019) : 547-553. https://doi.org/10.35940/ijitee.I1086.0789S19 